\title{
COMMISSION 12: SOLAR RADIATION AND STRUCTURE
}

\section{(RADIATION ET STRUCTURE SOLAIRES)}

\author{
PRESIDENT: Peter Foukal \\ VICE-PRESIDENT: Sami Solanki \\ ORGANIZING COMMITTEE: J. Mariska (Secretary), S. Baliunas, D. Dravins, \\ T. Duvall, C. Fang, V. Gaizauskas, P. Heinzel, E. Kononovich, S. Koutchmy, \\ D. Melrose, M. Stix, Y. Suematsu, \& F. Deubner
}

\section{INTRODUCTION}

The focus of Commission 12 is on the solar interior, on global phenomena of the quiet Sun, and on the Sun's radiative outputs, both spectral and total. These are the topics treated below in our discussion of Scientific Highlights. The many topics having to do with solar activity at photospheric to coronal levels, are dealt with in the report by Commission 10, while the report of Commission 49 describes research on the solar wind and interplanetary medium.

As remarkable as the scientific advances in our understanding of the Sun, is the wide range of innovative instruments, data bases and techniques used to obtain these results. These include huge neutrino detectors located deep below the Earth's surface, sophisticated space-borne telescopes, spectrometers, and radiometers, global networks of telescopes making intricately coordinated observations, and also extend to the reconstruction of past solar behavior from archival solar measurements and from paleo-climatological data. Some of these instruments and techniques still await full exploitation, so equal or greater riches of increased understanding await us in the next triennium.

I thank F. Deubner, T. Duvall, J. Mariska, J. Pasachoff, S. Solanki, and M. Stix for providing much of the material in this summary.

\section{SCIENTIFIC HIGHLIGHTS}

The past triennium has seen important advances in our understanding of the solar interior, provided in large part from a variety of helioseismic measurements mentioned below, but also from improved physical data on opacities (e.g. Berrington 1997) and on nuclear reaction rates (see e.g. review by Adelberger et al. 1998).

New models based on the helioseismic data by e.g. Takata and Shibahashi (1998) have provided, for one, an accurate determination of the helium content ( $y=0.248)$ in the outer solar layers (Richard et al. 1998). They have also yielded a much more accurate location of the base of the solar convection zone, at $r=0.713 \pm 0.001 \mathrm{R}_{\odot}$, from the Sun's center (Basu and Antia 1997), as well as a new determination of the Sun's radius (Schou et al. 1997, Antia 1998). They also show that the gravitational constant, G, is constant in time to better than $10^{-12}$, thus to higher accuracy even than pulsar timings can provide (Guenther et al. 1998).

An important consequence of the improved precision of the standard solar model is to confirm that the discrepancy between predicted and observed solar neutrino luminosity cannot be resolved through uncertainties of the solar interior model, so the solution must be sought in neutrino oscillations (see e.g. the review by Castellani et al. 1997). Additional neutrino measurements such as those from the Super-Kamiokande detector confirm and elucidate the discrepancy. 
Helioseismic data have also clarified the kinematics of the Sun's interior. They show that the angular velocity in the convection zone follows the same latitude dependence (i.e. equatorial acceleration) as the photosphere. The radiative core, on the other hand, rotates uniformly. A transition layer of radial shear of thickness approximately $0.1 \mathrm{R}_{\odot}$, separates these two rotational regimes.

Additionally, helioseismic data analyzed by three different techniques now resolve the uncertainties that have long surrounded the reality of meridional flows in the solar atmosphere. These new data show that a poleward flow exists in at least the outer $10 \%$ of the solar radius (Giles et al. 1997, 1998; Schou and Bogart, 1998; Gonzalez Hernandez et al. 1998; Braun and Fan, 1998).

Interesting evidence for a torsional oscillation (i.e. a perturbation in solar rotational velocity propagating toward the equator during a solar cycle), obtained earlier from Doppler measurements of photospheric rotation has now been confirmed by helioseismic data, the oscillation has been measured to a depth of at least $0.05 \mathrm{R}_{\odot}$, and also to lower latitudes than seen in the earlier photospheric data (Schou, 1999).

Important advances have also been made in demonstrating the feasibility of threedimensional tomography of plasma flows, and of wave speed inhomogeneities in near-surface layers (e.g. Duvall et al. 1997; Kosovichev and Duvall, 1997). Such techniques should provide interesting insights into thermal structure and motions around magnetic active regions, for instance. But detailed results remain controversial.

New constraints on the amount of mechanical energy available for heating the quient chromosphere and corona are emerging from recent computations of chromospheric p-mode resonances (Steffens, 1998) and from helioseismic observations (Deubner et al. 1996; Jefferies et al. 1997).

Passing now to the topic of solar abundances, more detailed studies have been made of the interesting earlier result that transition region plasmas are enriched by about a factor two relative to photospheric abundances for elements that have first ionization potentials (FIP) below $10 \mathrm{eV}$ (Feldman and Widing 1993). This is true of both quiet Sun and coronal holes. Coronal plasmas turn out to be enriched by about a factor four, for the same lowFIP elements. In contrast, the abundance of high FIP ( $>11.5 \mathrm{ev)} \mathrm{elements} \mathrm{in} \mathrm{both} \mathrm{the}$ transition region and corona are the same as in the photosphere (Young and Mason, 1998).

Another advance is in our understanding of the morphology of the plasma in the transition regime between chromospheric $\left(10^{4} \mathrm{~K}\right)$, and coronal $\left(10^{6} \mathrm{~K}\right)$ temperatures, which has been refined by imaging with the SUMER instrument on the SOHO spacecraft, and also by the TRACE experiment. The SUMER images show that most of the emission in the $30,000 \mathrm{~K}-700,000 \mathrm{~K}$ temperature range originates in loop-like structures about 10 " long with widths below the 1.5" spatial resolution. These loops are concentrated at cell boundaries and oriented transverse to them, with a smaller number of fainter loops originating in cell interiors (Lemaire et al. 1997; Feldman et al. 1999).

Useful advances have also been made in our understanding of time variations in the solar total and spectral irradiances, and of their potential impact on climate change. The full 20-year record of satellite pyrheliometry has been placed on a common radiometric scale to provide a clearer picture of total irradiance variations on time scales from days to the past two solar activity cycles (Frohlich and Lean 1998). The main result is that most of the variance in this record can be accounted for by variations in the position and projected area of dark Sunspots and bright faculae in active regions and in associated enhanced network. There is no significant evidence for other mechanisms of solar luminosity variations over this period, at least.

The first long-term record of the areas of bright magnetic facular elements that dominate solar total and ultraviolet irradiance variation, was obtained from digitization of the Mt. Wilson archive of daily Ca K images extending to 1905 (Foukal 1996). Empirical models based on these data (Foukal, 1998) indicate that UV irradiances at the wavelengths that determine stratospheric ozone concentrations, were $20-30 \%$ higher in the 1915 to 1945 period than expected from previous rough estimates based on the Sunspot number and 
on various proxies of facular area. Fligge et al. (1998) presented a comprehensive, semiempirical model of solar spectral variability, which will serve to promote further work in this important area.

Results from empirical modeling of total and UV solar irradiances were used by Lean et al. (1995) to make the interesting point that the correlation of the climate record with solar activity is highest in the pre-industrial era, before 1800 , indicating a dominant effect of the Sun's variation on climate. But this correlation declines in the recent era, accounting for no more than half of the most rapid global warming, since 1860 .

\section{WORKING GROUPS}

The Working Group on Eclipses consists of Jay M. Pasachoff (Williamstown, Massachusetts) as Chair, Iraida Kim (Moscow), H. Kurokawa (Kyoto), Jagdev Singh (Bangalore), V. Rusin (Tatranska Lomnica), Fred Espenak (NASA-Goddard), Jay Anderson (Winnipeg), and Frederic Clette (Bruxelles). During the period since the Kyoto IAU General Assembly, the total eclipses were on 9 March 1997 in Mongolia and China, on 26 February 1998 in northern South America and the Caribbean, and on 11 August 1999 across Europe, Turkey, Syria, Iran, Iraq, Pakistan, and India. Espenak and Anderson continue to put out a series of NASA Reference Publications for each total eclipse. Espenak makes available this information as well as information about annular and partial eclipses on his Web site, http://sunearth.gsfc.nasa.gov/eclipse/eclipse.html.

The Working Group maintains a web site at http://williams.edu/astronomy/iau_eclipses. This site contains descriptions of scientific expeditions, and before the eclipse, liaison information. Such information includes contacts for customs importation of scientific equipment. The site also includes information about viewing eclipses safely. The Working Group responds to requests about safe viewing of eclipses, with Dr. Ralph Chou, a professor of optometry, as a consultant. It is clear that hundreds of millions of people around the world are confused about the distinction between partial and total eclipses, and we suggest that this point be emphasized in future publicity.

The next total eclipses will be on 21 June 2001 in southern Africa and on 4 December 2002, again in southern Africa but ending at sunset in southern Australia.

\section{References}

Adelberger, E.G., Austin, S.M., Bahcall, J.N., and 36 others (1998): Rev. Mod. Phys. 70, 1265.

Antia, H.M. (1998): Astron. Astrophys. 330, 336.

Basu, S., Antia, H.M. (1997): Mon. Not. R. Astron. Soc. 287, 189.

Berrington, K.A. (ed.) (1997): The Opacity Project, Vol. 2, Institute of Physics, Bristol and Philadelphia.

Braun, D.C. and Fan, Y., 1998, ApJ, 508, L105-L108.

Castellani, V., Degl'Innocenti, S., Fiorentini, G., Lissia, M., Ricci, B. (1997): Physics Reports 281, 309.

Deubner, F., Waldschik, T., and Steffens, S., 1996, Astr. Ap., 307, 936.

Duvall, Jr., T.L., Kosovichev, A.G., Scherrer, P.H., Bogart, R.S., Bush, R.I., De Forest, C., Hoeksema, J.T., Schou, J., Saba, J.L.R., Tarbell, T.D., Title, A.M. Wolfson, C.J., Milford, P.N., 1997, Solar Physics 170, 63-73.

Feldman, U. and Widing, K.G. 1993 ApJ, 414, 381.

Feldman, U. and Widing, K.G., and Warren, H.P. 1999 ApJ 522, 1133.

Fligge, M. Solanki, S., Unruh, X., Frohlich, C., and Wehrli, C. 1998, Ast. Ap. 335, 709.

Foukal, P. 1996, Geophys. Res. Letts. 23, 2169.

Foukal, P. 1998, Geophys. Res. Letts. 25, 2909. 
Frohlich, C. and Lean, J. GRL 25, 4377.

Giles, P.M., Duvall Jr., T.L., Scherrer, P.H., and Bogart, R.S., 1997 Nature 390, 52-54.

Giles, P.M., Duvall Jr., T.L., and Scherrer, P.H., 1998, in Structure and Dynamics of the Sun and Sun-Like Stars, eds. S.G. Korzennik and A. Wilson, 775-780.

Gonzalez Hernandez, I., Patron, J., and Bogart, R.S., 1998, in Structure and Dynamics of the Sun and Sun-Like Stars, eds. S.G. Korzennik and A. Wilson, 587-595.

Guenther, D., Krauss, L., and DeMarque, P., 1998, Ap.J. 498, 871.

Jefferies, S., Osaki, Y., Shibahashi, H., Harvey, J., D'Silva, S., and Duvall, T., 1997, ApJ, $485, \mathrm{~L} 49$.

Junker, M., D'Alessandro, A., Zavaterlli, S., and 16 others (1998): Phys. Rev. C 57, 2700.

Kosovichev, A.G. and Duvall, T.L. Jr., in F. Pijpers et al. (eds.), SCORe '96: Solar Convection and Oscillations and their Relationship, Kluwer Academic Publishers, Dordrecht, Holland, 241-260 (1997).

Kosovichev, A.G., Schou, J., Scherrer, P.H., and 31 others (1997): Solar Phys. 170, 43.

Lean, J., Beer, J., and Bradley, R., 1995, Geophys. Res. Letts. 22, 3195.

Lemaire, P. et al. 1997 Solar Phys., 170, 105.

Richard, O., Dziembowski, W.A., Sienkiewicz, R., Goode, P.R. (1998): Astron. Astrophys. 489, L197.

Schou, J. and Bogart, R.S., 1998 ApJ 504, L131-L134.

Schou, J., 1999 Ap.J. 523, L181.

Schou, J., Kosovichev, A., Goode, P. Dziembowski, W. 1997 Ap.J. 489, L197.

Steffens, S., 1998, PhD Thesis, Würzburg Univesity.

Takata, M., Shibahashi, H. (1998): Astrophys. J. 504, 1035.

Young, P.R., and Mason, H.E. 1998 Space Sci, Rev., 85, 315.

\section{SOURCES OF FURTHER INFORMATION}

Further information on advances in research on the solar interior is available on the web at http://helios.tuc.noao.edu/new.html and also at http://soi.stanford.edu/\#news. Further information on Division II activities can be obtained from the Division II page on the IAU website http://www.iau.org.

Peter V. Foukal

President of the Commission 